\title{
CT colonography: avoiding traps and pitfalls
}

\author{
Philippe Lefere $\cdot$ Stefaan Gryspeerdt
}

Received: 5 August 2010/Accepted: 18 November 2010 /Published online: 4 January 2011

(C) European Society of Radiology 2010

\begin{abstract}
Computed tomographic colonography (CTC) is a reliable technique for detecting tumoral lesions in the colon. However, good performance of polyp detection is only achieved if experienced CTC radiologists combine meticulous interpretation with state-of-the-art CTC technique. To reach this experience level, CTC training is mandatory. With a considerably long and steep learning curve, it has been demonstrated that in inexperienced hands both technical failure and observer errors stand for the majority of missed lesions. The purpose of this pictorial review is to give an overview of traps and pitfalls in CTC imaging resulting in false negative and positive findings, and how to avoid them by application of state-of-the-art CTC technique and interpretation.
\end{abstract}

Keywords Computed tomographic colonography .

CTC $\cdot 2 \mathrm{D} \cdot 3 \mathrm{D} \cdot$ Pitfalls

\section{Introduction}

Computed tomographic colonography (CTC) is a reliable technique for detecting tumoral lesions in the colon [1-4]. However, good performance in polyp detection is only achieved if experienced CTC radiologists combine meticulous interpretation with state-of-the-art CTC technique [5]. To reach this experience level, CTC training is mandatory. With a considerably long and steep learning curve, it has been demonstrated that in inexperienced hands both

P. Lefere $(\triangle) \cdot$ S. Gryspeerdt

VCTC-Virtual Colonoscopy Teaching Centre,

Akkerstraat 32c,

8830 Hooglede, Belgium

e-mail: info@vctc.eu technical failure and observer errors account for the majority of missed lesions [6,7]. The purpose of this pictorial review is to give an overview of traps and pitfalls in CTC imaging resulting in false-negative and falsepositive findings, and how to avoid them by application of state-of-the-art CTC technique and interpretation.

Pitfalls in imaging are related to the technical aspects of CTC, the anatomical aspect of the colon and two- (2D) and three-dimensional (3D) imaging.

\section{Technique-related pitfalls}

Preparation

CTC requires the colon to be as clean and dry as possible for adequate interpretation [8]. Together with preparation combining a low residue diet with laxatives or cathartics, faecal tagging is an essential part of this process [9]. By impregnating the faecal residue, stool tagging not only allows for differentiation between solid residue and a true polypoid lesion (Fig. 1), fluid tagging also enables detection of polyps as negative filling defects in tagged fluid (Fig. 2).

Even if these requirements for preparation are fulfilled, problems may still occur with non-tagged stool mimicking a polypoid lesion.

Using dual positioning (= supine/prone acquisition) and 2D imaging most problems are solved, as the stool can be correctly identified in the case of:

A luminal defect with air inclusion: mottled appearance Positional shift (Fig. 3)

A defect floating in a pool of barium (Fig. 3)

A defect with a hyperdense peripheral ring and a hypodense centre 


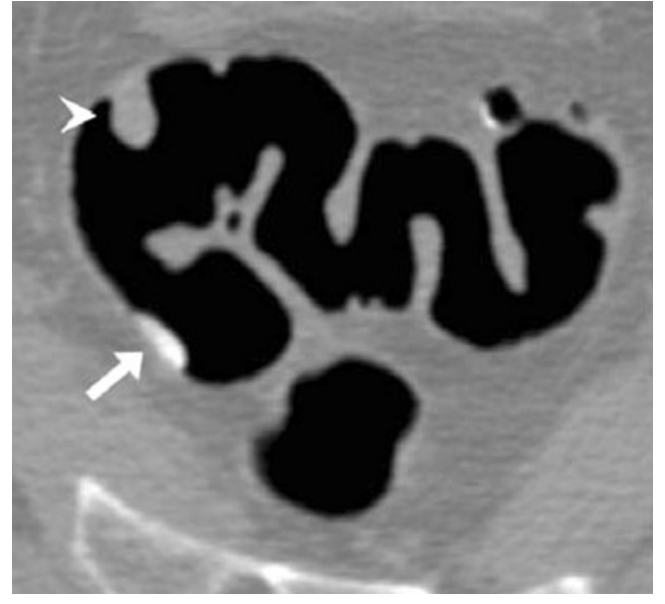

Fig. 1 Ascending colon: sessile 9-mm polyp with soft tissue density (white arrowhead) in contrast material with tagged residue on the posterior wall (white arrow)

In about $50 \%$ of cases tumoural lesions are covered by contrast material causing an aspect of heterogeneous tagging [10]. Correct identification can be obtained by comparing the supine and prone acquisitions and by appreciating the typical characteristics of the tumoural lesions beneath the contrast material layer (Fig. 4).

\section{Distension}

Optimal colonic distension is the second cornerstone of good CTC practice. This is achieved by combining smooth muscle relaxation, colonic inflation with carbon dioxide (preferably with a $\mathrm{CO}_{2}$ injector) and dual positioning. Using a $\mathrm{CO}_{2}$ injector allows monitoring the pressure in the colon. It is important to keep pressure in the colon between 20 and $25 \mathrm{mmHg}$. Besides this, it is very important to wait until the patient mentions abdominal problems such as bloating. Tips to avoid particular problems will be given in the anatomy section. One particular entity needs our attention: pneumatosis coli [11]. This is an uneventful benign complication of the colonic insufflation. It presents as submucosal and/or subserosal air bubbles or cysts with a polypoid appearance in $3 \mathrm{D}$. In $2 \mathrm{D}$, the diagnosis of pneumatosis coli is made straightforward by identifying the air collections (Fig. 5).

\section{Acquisition}

\section{Dose-related problems}

CTC is particularly well designed for low-dose acquisition because of the high contrast between the luminal gas and the colonic wall. In some cases a low dose causes excess image noise resulting in pseudo-enhancement: an artificial increase in the tissue density sometimes resulting in pseudo-tagging, i.e. making a true lesion look like tagged stool (Fig. 6) [12]. This problem can be solved by comparing the lesion with tagged stool elsewhere in the colon or by smoothing the abdominal window. When starting CTC as a novice, a dose of $80 \mathrm{mAs}$ should be used. This avoids image noise. With growing experience the dose can be decreased to $50 \mathrm{mAs}$. Ideal for $\geq 64$-slice systems, this dose produces considerable noise in 16-slice systems.

\section{Motion artefacts}

Although recent systems allow for very short acquisition times and require only a short breath-hold, motion artefacts may deteriorate image quality or cause pseudo-lesions because of:

- A "twin fold" aspect: two longitudinal defects close to each other (Fig. 7)
Fig. 2 a Ascending colon, axial view: thickened fold in tagged fluid (white arrowhead).

b Corresponding coronal image shows a 12-mm lesion with flat morphology as a negative filling defect in the tagged fluid (black arrowhead)
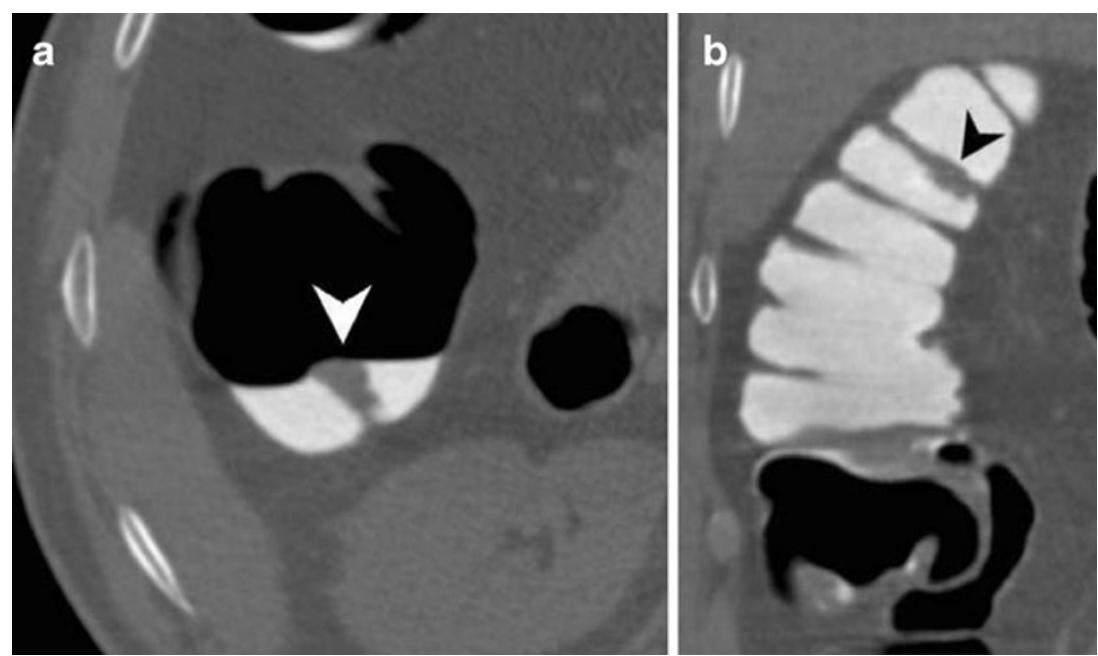

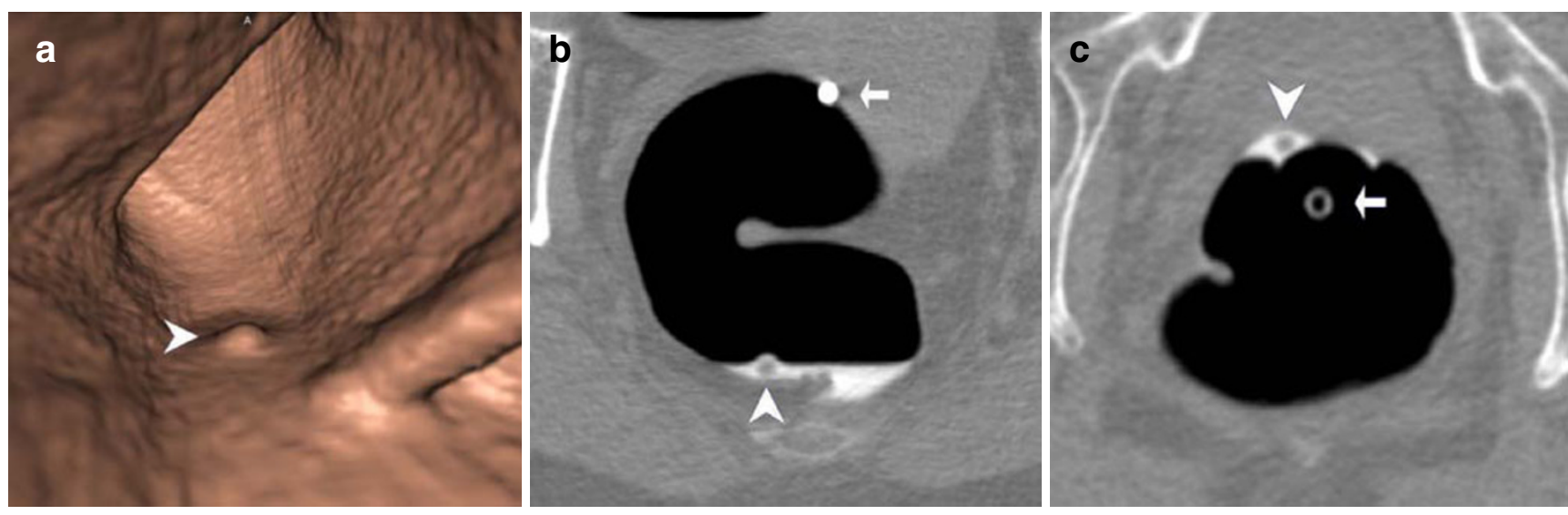

Fig. 3 a Three-dimensional view of the rectum showing a small polypoid defect surrounded by some fluid (white arrowhead). b The corresponding axial image in the supine position shows a negative filling defect floating in a contrast material pool (white arrowhead).

- Curved or serpiginous appearance of a fold

- Pseudopolypoid aspect

Motion artefacts can be confirmed in 2D by looking for stair steps on the abdominal wall on the sagittal reformats. As a general rule it is mandatory to always compare $3 \mathrm{D}$ with $2 \mathrm{D}$ when detecting a mural abnormality (Fig. 7c).

\section{Anatomy-related pitfalls}

General remarks

Some pitfalls occur in any of the colonic segments.

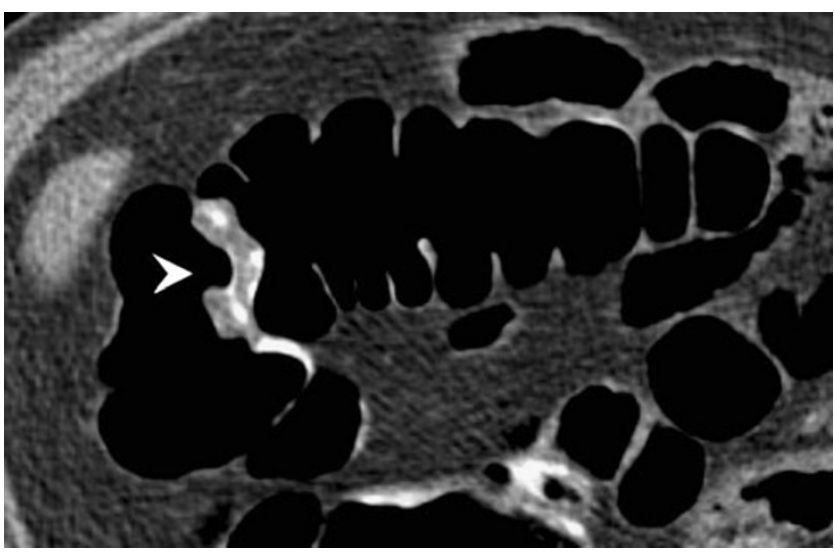

Fig. 4 Axial view of the hepatic flexure showing a large defect with solid components and contrast material (white arrowhead). Image of large tumoural lesion covered by contrast material
White arrow hyperdense tip of the rectal catheter. c In the prone position the filling defect has moved to the anterior part of the rectum (white arrowhead). Both positional shift and floating of the defect in contrast material allow for a correct diagnosis of non-tagged stool

\section{Segmental mobility}

Due to a long mesentery, some segments of the colon frequently change in position with dual positioning [13]. This occurs in all segments and more specifically at the level of all flexures, the sigmoid, the transverse colon and the caecum. These movements are variable: turning over its longitudinal axis (flipping), rotating or more complex movements. They create an aspect of pseudo-stool as polypoid lesions change in position together with the moving segment. Comparing the 2D images in the different reformats and referring to other structures such as the ileocaecal valve, diverticula and diverticular faecaliths allows for detecting the positional change of the colonic segment (Fig. 8).

\section{Flexural pseudo-tumour}

At each flexure of the colon, pseudo-thickening of the colonic wall at the inner side of the flexure may occur because of compression of the colonic wall. This is sometimes accentuated by the pericolonic fat and/or vascular structures. This phenomenon is called the flexural pseudo-tumour [14]. In 3D a thickened fold is detected sometimes with a pseudo-polypoid aspect. Frequently there is also convergence of multiple folds. The thickened fold has a smooth appearance and mostly changes in shape with dual positioning. In $2 \mathrm{D}$ a thickened fold situated at the inner part of a flexure is shown (Fig. 9).

\section{Extrinsic impressions}

Extra-colonic structures occur anywhere in the colon. They are more frequent when colonic distension is optimal. They 
Fig. 5 a Three-dimensional view of the splenic flexure reveals multiple polypoid defects. Some present with a shine-through phenomenon (white arrows).b The corresponding axial image shows submucosal (white arrows) and subserosal (white arrowhead) air collections consistent with pneumatosis coli
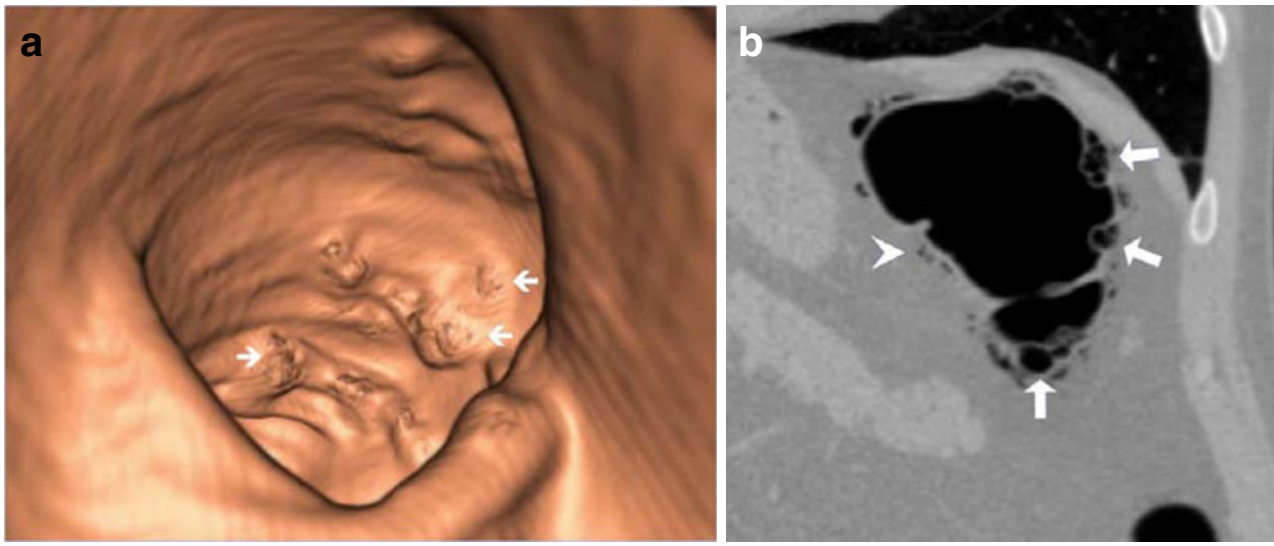

may be caused by normal or pathological structures. In 3D they sometimes mimic a polypoid structure. Again, correlation with the corresponding $2 \mathrm{D}$ images is mandatory to detect the origin of the 3D bump (Fig. 10).

\section{Rectum}

The rectum is particularly difficult to examine.

\section{Anal margin}

The rectum is a closed structure ending at the anal margin. Tumoural lesions may be located at the anal margin. It is mandatory to inspect the anal margin corresponding to the region where the rectal catheter enters the rectum. Peri-anal lesions may be true tumoural lesions. They are to be differentiated from a hypertrophied anal papilla. CTC cannot differentiate between the two entities and once a lesion measures $\geq 6 \mathrm{~mm}$, optical colonoscopy is necessary to rule out a significant lesion (Fig. 11).

Internal haemorrhoids represent another cause of pseudopolypoid lesion close to the anal margin. They look like broad-based, grape-like defects surrounding the catheter. They usually change in shape with dual positioning [15].
Frequently, longitudinal folds converging on the anal canal are present. The most prominent or rectal bar is located anteriorly and may appear as a polyp on $2 \mathrm{D}$ images. In 3D this is clearly depicted it as a fold.

\section{Rectal ampulla}

The rectal ampulla is the segment of the rectum above the anal canal. It is the widest portion, making it more difficult to cover its entire wall. Furthermore, some parts may be hidden by the rectal catheter. A combined 2D-3D approach using coronal and sagittal images is to be considered. Detailed inspection with 3D requires the widest angle of the virtual camera: $\geq 120^{\circ}$.

\section{Rectal valves}

The rectal valves or valves of Houston, mostly three (inferior, middle, superior) are prominent transverse folds supporting the weight of the faecal matter [16]. As they are prominent, they may hide lesions and need special attention by using the $120^{\circ}$ camera angle, by looking well behind them and relying upon coronal and sagittal images.
Fig. 6 a Ultra-low dose image $(140 \mathrm{kV}, 10 \mathrm{mAs})$ of the sigmoid: pedunculated polyp with increased density due to image noise (pseudoenhancement) (white arrow). b Same patient imaged with a normal dose for staging of malignant tumour elsewhere in the colon. The same polyp shows a normal soft tissue density (white arrow)
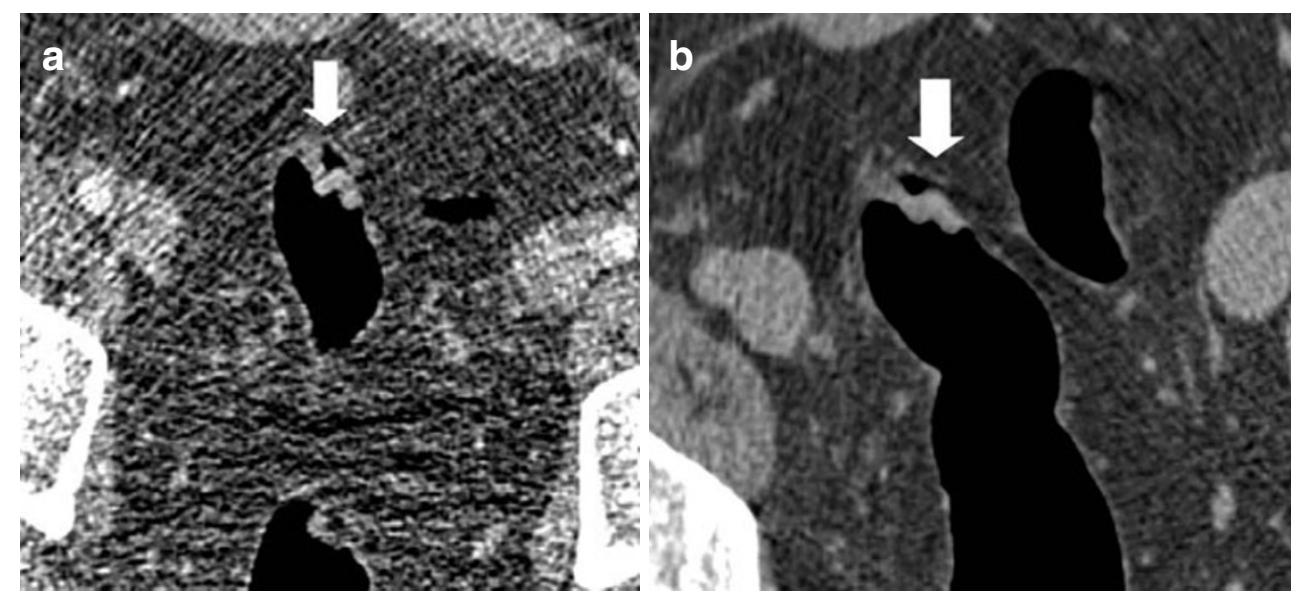

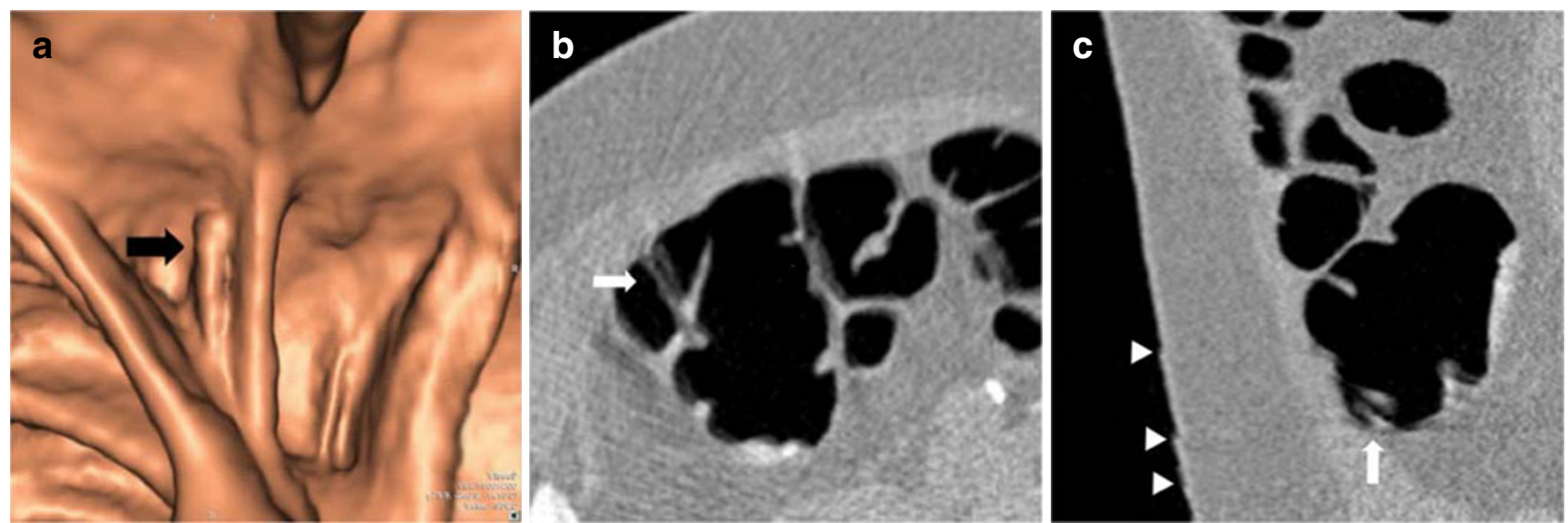

Fig. 7 a Three-dimensional view of the ascending colon showing a longitudinal luminal defect along a semi-lunar fold (black arrow): flat lesion? b, c The corresponding axial and sagittal views show a subtle stair step artefact on the abdominal wall (white arrowheads) and a doubled or "twin fold" aspect of the semilunar fold explaining the small longitudinal defect as a subtle breathing artefact

\section{Rectal distension}

The rectum is situated posteriorly in the pelvis, making adequate distension in the supine position arduous and optimal distension in the prone position easy. The basic principles of distension, as mentioned above, need special attention.

\section{Rectal tube}

The rectal tube may compromise good and/or the entire visualisation of the rectum and hence obscure a lesion. Having a good look around the rectal catheter, where it abuts the rectal wall, is mandatory. As it is for rectal use, the catheter is provided with an inflatable balloon prevent-
Fig. 8 a Axial view of the caecum in the supine position showing an 8-mm polyp on the left lateral side (white arrowhead). b The corresponding view in the prone position shows the same defect on the anterior caecal wall, submerged in slightly tagged fluid (white arrowhead). Residual stool with positional shift? c, d There is a rotation of the caecum over $90^{\circ}$ as is shown by the different positions of the ileo-caecal valve (white arrowhead) and the terminal ileum (white arrow) in both acquisitions: image of true polyp or pseudo-stool due to segmental mobility
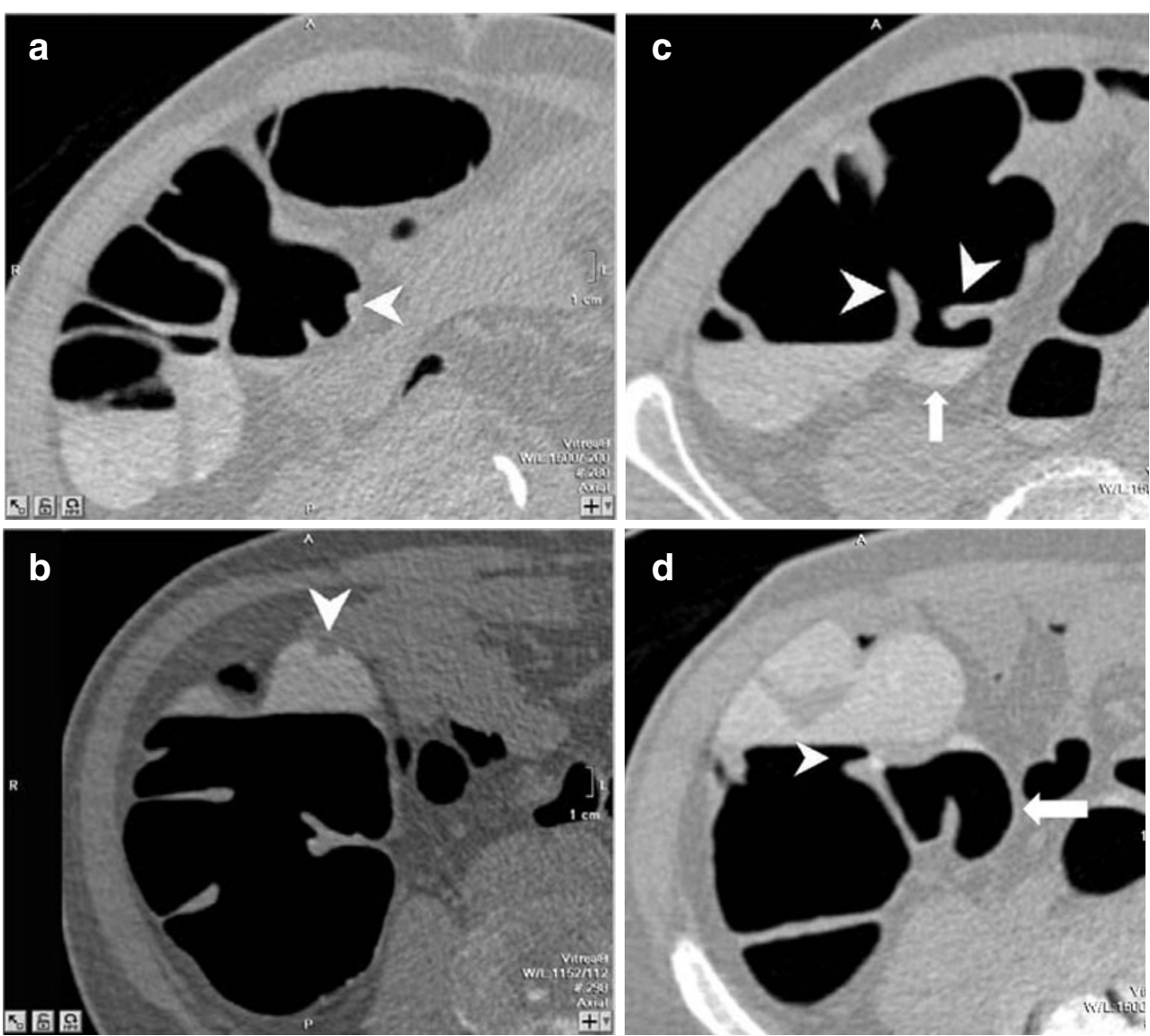

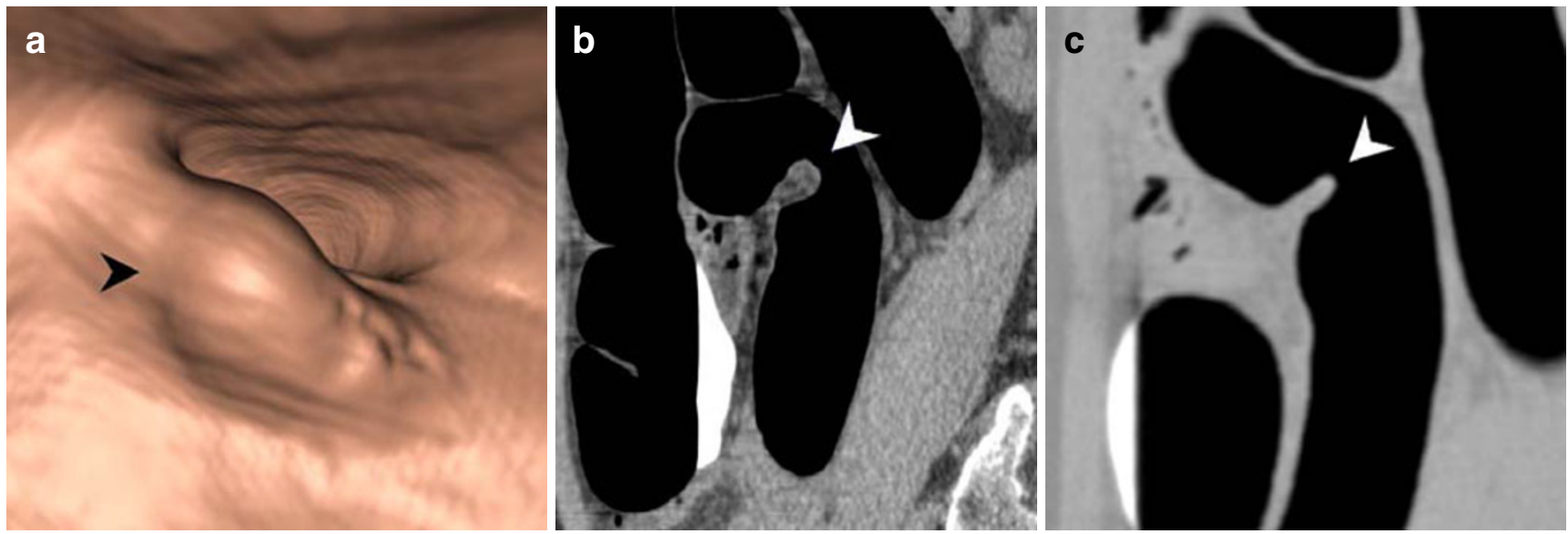

Fig. 9 a Three-dimensional view of the sigmoid in a patient with dolichocolon: image suggestive of large polypoid mass (black arrowhead). b Corresponding sagittal view in an abdominal window setting showing an acute flexure of the sigmoid over $180^{\circ}$ with thickening of the colonic wall and compression of the inner structures. c The corresponding 3D view in the prone position shows a normal fold

images. Do not mistake a polyp with serpiginous aspect for a rectal varix.

\section{Sigmoid}

\section{Distension}

The taenia coli are three longitudinal muscular bands, starting in the sigmoid and converging at the appendicular orifice in the caecum and define the appearance of the colonic lumen by their contraction status [17]. With increased contraction, the haustral folds located between the semilunar folds become deeper, making inspection of the colonic wall more arduous. In the sigmoid, they are not prominent and give a round or oval shape to the sigmoidal
Fig. 10 a Three-dimensional view at the hepatic flexure showing a tumoural mass with overhanging edges (black arrow). There is a subtle bump on the colonic wall more proximally (white arrow). b The corresponding 2D image shows the large tumour with overhanging edges and shoulder formation (black arrow). The subtle bump was caused by a small pericolonic nodule (white arrow) suggestive of mesenteric metastasis
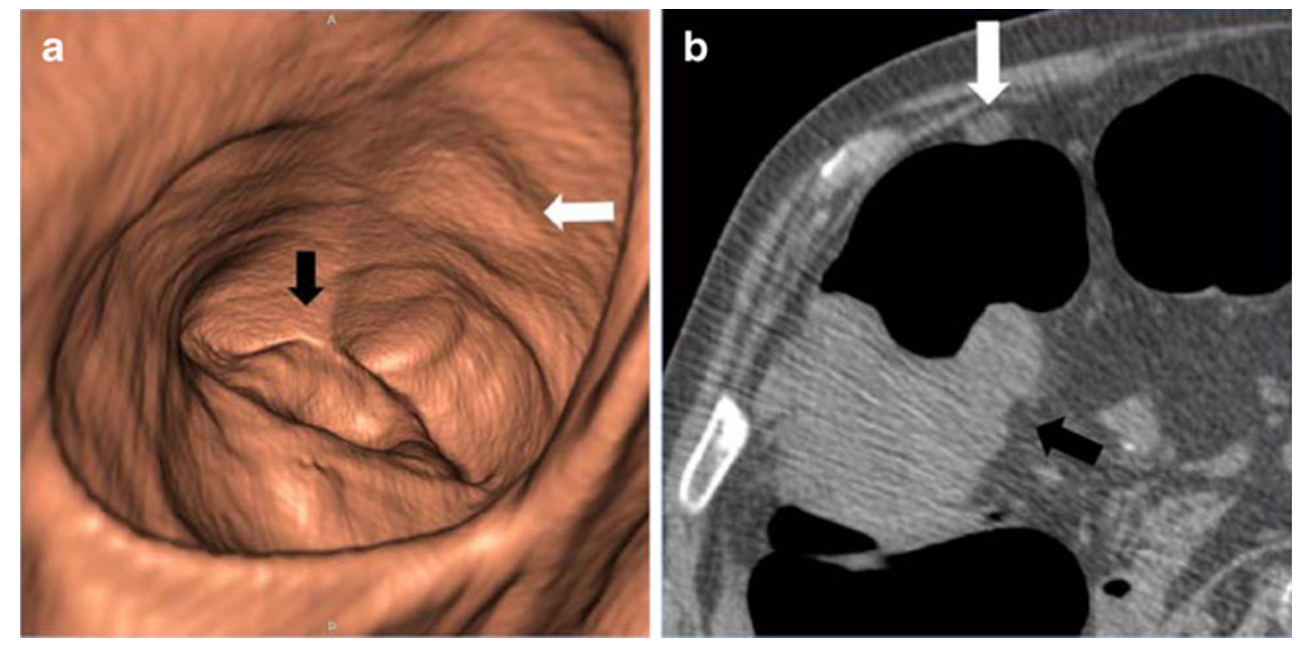


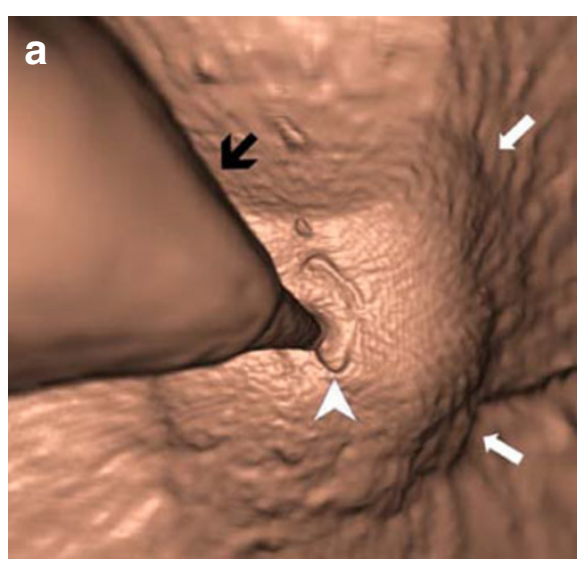

Fig. 11 a The rectum and its "peri-catheter" segment need special attention! Three-dimensional view of the anal margin shows interpretation is hampered by the rectal catheter (black arrow). The impression of the inflated balloon on the rectal wall is nicely appreciated (white arrows). A flat luminal defect is detected at the level of the anal margin, abutting the rectal catheter (white arrowhead). b, c The corresponding prone view after deflation of the balloon of the rectal catheter shows a large sessile polypoid defect (white arrowhead) against the rectal catheter (black arrow) confirmed on $2 \mathrm{D}$ as a solid lesion $>10 \mathrm{~mm}$, prompting optical colonoscopy lumen. In the case of spasm the sigmoidal lumen shows a round narrowing with smooth, slightly thickened folds. On 2D CTC, a spasm may appear as kissing folds: two thickened folds touching each other.

\section{Diverticulosis}

In Western countries, diverticulosis is the most common colonic disease and harbours many traps in colonic imaging with CTC. Because of myochosis there is reduced distension of the sigmoid, making the semilunar folds more prominent with restricted visualisation of the sigmoidal lumen. Sometimes an additional right/left decubitus acquisition is required to obtain optimal distension [18]. It is obvious that diverticulosis is not restricted to the sigmoid and that the imaging characteristics as described below may appear anywhere in the colon.

\section{Diverticulum}

In $3 \mathrm{D}$ the diverticulum may mimic a polypoid lesion. Mostly it presents as a completely dark ring, compared with the incomplete dark ring of the polyp when viewed en face. In $2 \mathrm{D}$ the diverticular outpouchings are clearly visualised [19].

\section{Diverticular faecalith}

A diverticulum may become impacted with residual stool. As the diverticulum lacks a muscular layer, the stool is not expelled, remains in the diverticulum, dries and hardens into a faecalith [19]. In 3D it frequently presents as a polypoid image, while in 2D it has a typical appearance and presents as a luminal defect with a hypodense centre and a hyperdense peripheral ring (Fig. 12). Whenever a luminal
Fig. 12 a Three-dimensional view of the sigmoid showing a polypoid defect (white arrow). b The corresponding axial image shows that the defect is caused by a nodular structure consisting of a hypodense centre and peripheral hyperdense ring typical of a diverticular faecalith (white arrow)
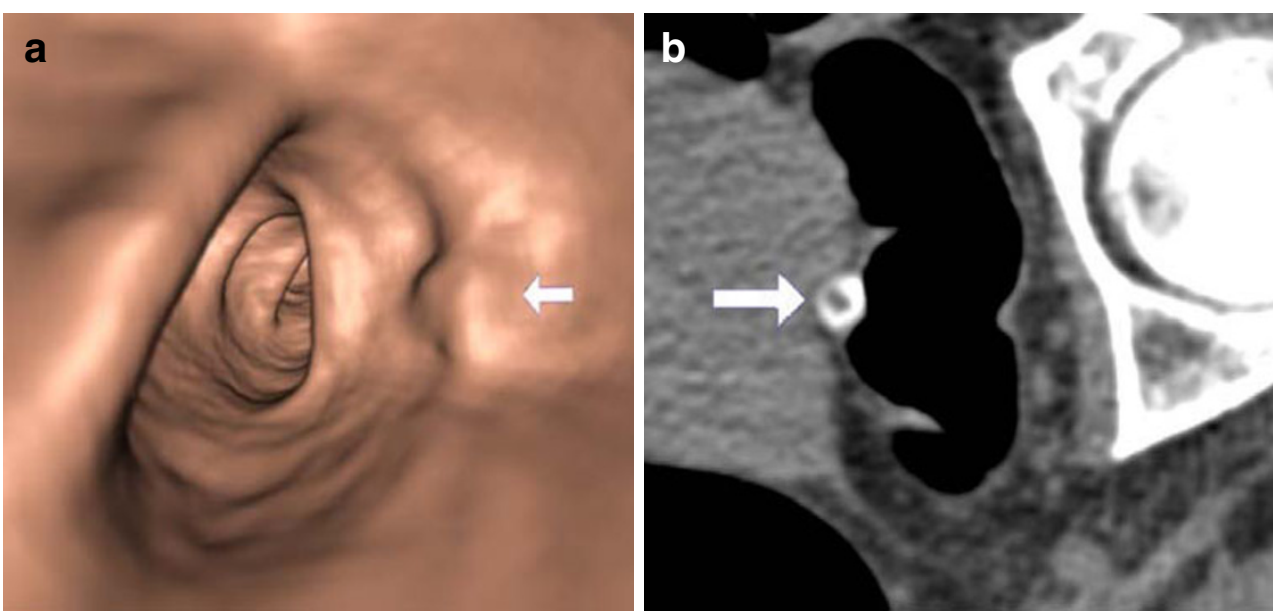
defect is encountered in $3 \mathrm{D}$, characterisation has to be performed with $2 \mathrm{D}$. If there are too many faecaliths, 3D becomes time-consuming and it is better to switch to primary 2D read. Exceptionally the diverticular faecalith inverts into the colonic lumen.

\section{Inverted diverticulum}

Although rare, wherever a diverticulum appears it may invert into the colonic lumen [20]. Three-dimensional CTC shows a sessile polypoid lesion. As the diverticulum usually takes some luminal air or perisigmoidal fat when inverting, it presents in 2D with an air and/or fat inclusion. Again, comparison of the 3D and 2D findings is mandatory to come to the right diagnosis (Fig. 13).

\section{Wall thickening}

Chronic diverticulosis with periods of diverticulitis may result in wall thickening. Differential diagnosis with tumour is not always straightforward and this entity is known with confusion in $10-50 \%$ of cases with an exact diagnosis in one case out of two [21]. Table 1 shows the imaging characteristics of the two entities.

\section{Polypoid mucosal prolapse syndrome}

With progression of diverticular disease there is sometimes creation of an excess of mucosa because of further shortening and contraction of the muscular layer [22]. This mucosa prolapses into the colonic lumen and creates a polypoid image on both $2 \mathrm{D}$ and 3D. This pseudopolypoid image cannot be differentiated from a true polyp. So whenever its size exceeds $5 \mathrm{~mm}$, optical colonoscopy is needed. Sometimes the lesion changes in aspect with dual positioning. In that case, diagnosis can only be suggested and optical colonoscopy remains mandatory.

\section{Miscellaneous}

Metallic artefacts cause blurring of the structures with multiple streak artefacts, making interpretation in both $2 \mathrm{D}$ and 3D difficult. In 2D it is helpful in smoothing the window.

\section{Descending colon}

As the taenia coli becomes somewhat more prominent, the shape of the colonic lumen varies from tubular to slightly triangular. This shape is accentuated in case of spasm. Sometimes differentiation from a stenosing tumour is difficult. A tumour typically has overhanging edges, shoulder formation or may present with a bull's eye phenomenon (Fig. 10).

\section{Transverse colon}

The taenia coli are now really prominent, giving a typical triangular aspect to the colonic lumen. This is associated with deep haustrations. This necessitates good inspection of the colonic wall between the semilunar folds using a $120^{\circ}$ viewing angle of the virtual camera. In $2 \mathrm{D}$, coronal and sagittal images are helpful in achieving complete visualisation of the colonic wall. Again in the case of spasm, the narrowed segment presents usually with a triangular shape with smooth borders. Typically, kissing folds are distinguished (Fig. 14).

As the transverse colon is situated anteriorly in the abdomen, it tends to be compressed by the weight of the abdomen in the prone position. As important pathological features may be hidden, special care should be taken to
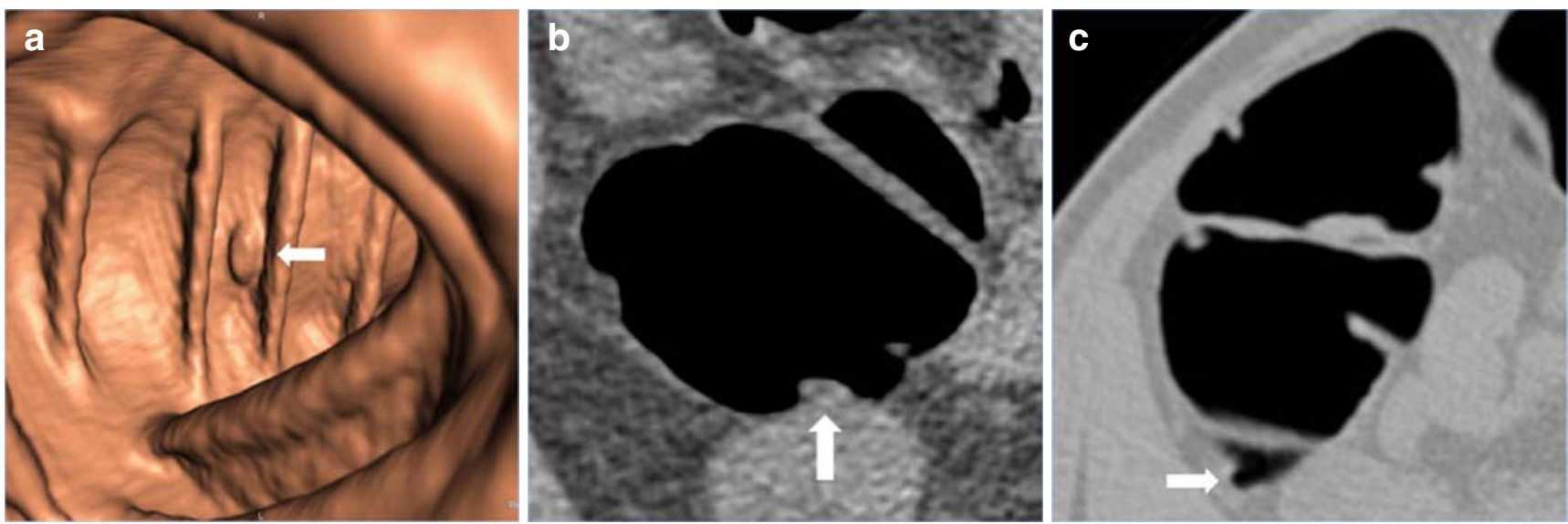

Fig. 13 a Three-dimensional view of the ascending colon in the supine position showing a 9-mm polypoid defect (white arrow). b The corresponding $2 \mathrm{D}$ view confirms the probable polyp (white arrow). $\mathbf{c}$ However, the prone acquisition reveals a diverticulum at the same location (white arrow). Review of the 2D image in the abdominal window shows a central hypodensity of -100 to $0 \mathrm{HU}$, consistent with a subtle amount of fat. These two findings confirm the diagnosis of inverted diverticulum 
Table 1 Differential diagnosis between fibrosis and adenocarcinoma. In the case of mild cone-shaped wall thickening with pericolonic stranding and without adenopathies, there is a $90 \%$ specificity for fibrosis. In the case of an apple core lesion or short wall thickening with shoulder formation and adenopathies specificity for a malignant tumour is $92 \%$

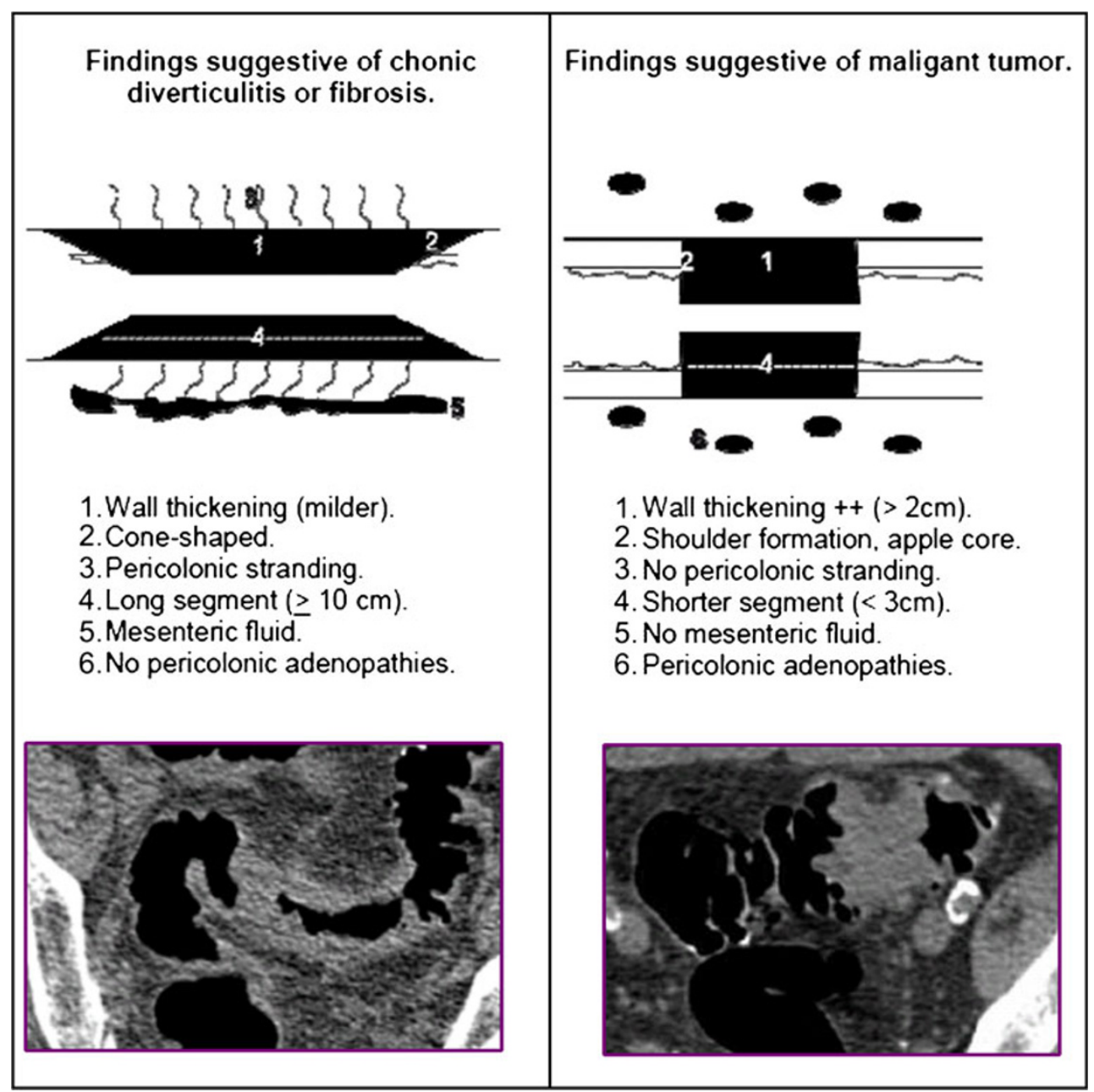

obtain good distension of the transverse colon in the prone position. Besides the requirements of good distension, it can be helpful putting a pillow under the breast to decrease pressure on the transverse colon (superman position). Examination of both acquisitions is of course mandatory.

\section{Ascending colon}

The taenia coli remain prominent, giving the typical triangular shape to the colonic lumen (Fig. 13).

\section{Caecum}

As with the rectum, the caecum is a closed structure or pouch needing special attention. Furthermore, the ileo-caecal valve and the appendicular orifice may render interpretation complicated. As the length of the caecum varies, it is frequently subject to flexural pseudo-tumour and to segmental mobility with caecum recurvatum internum and externum.

\section{Ileo-caecal valve}

It is mandatory to always localise the ileo-caecal (IC) valve when inspecting the caecum. The normal ileo-caecal valve presents as a "mouth-like" structure with an upper and a lower lip, converging in a larger fold, the frenulum. A central slit or opening represents the transition to the last ileal loop. This opening is always directed towards the caecal tip. Defining the normal morphology of the IC valve excludes tumoural pathological features and avoids mistaking the IC valve for a tumour. Comparing $3 \mathrm{D}$ with $2 \mathrm{D}$ images in both abdominal and intermediate window settings is again mandatory. Coronal and sagittal images are also helpful in assessing its structure. The IC valve has different aspects.

\section{- Lipomatous IC valve}

Infiltration with lipomatous tissue causes an enlargement of the IC valve without altering the normal structure. Diagnosis is 
Fig. 14 a, b Three-dimensional view of the transverse colon shows a luminal narrowing with a triangular shape: image typical of spasm caused by contraction of the taenia coli (white arrows). Thickened semilunar folds with a smooth aspect causing kissing folds on the corresponding axial image (white arrows). c Sagittal image showing the taenia coli (black arrows) with the triangular luminal narrowing
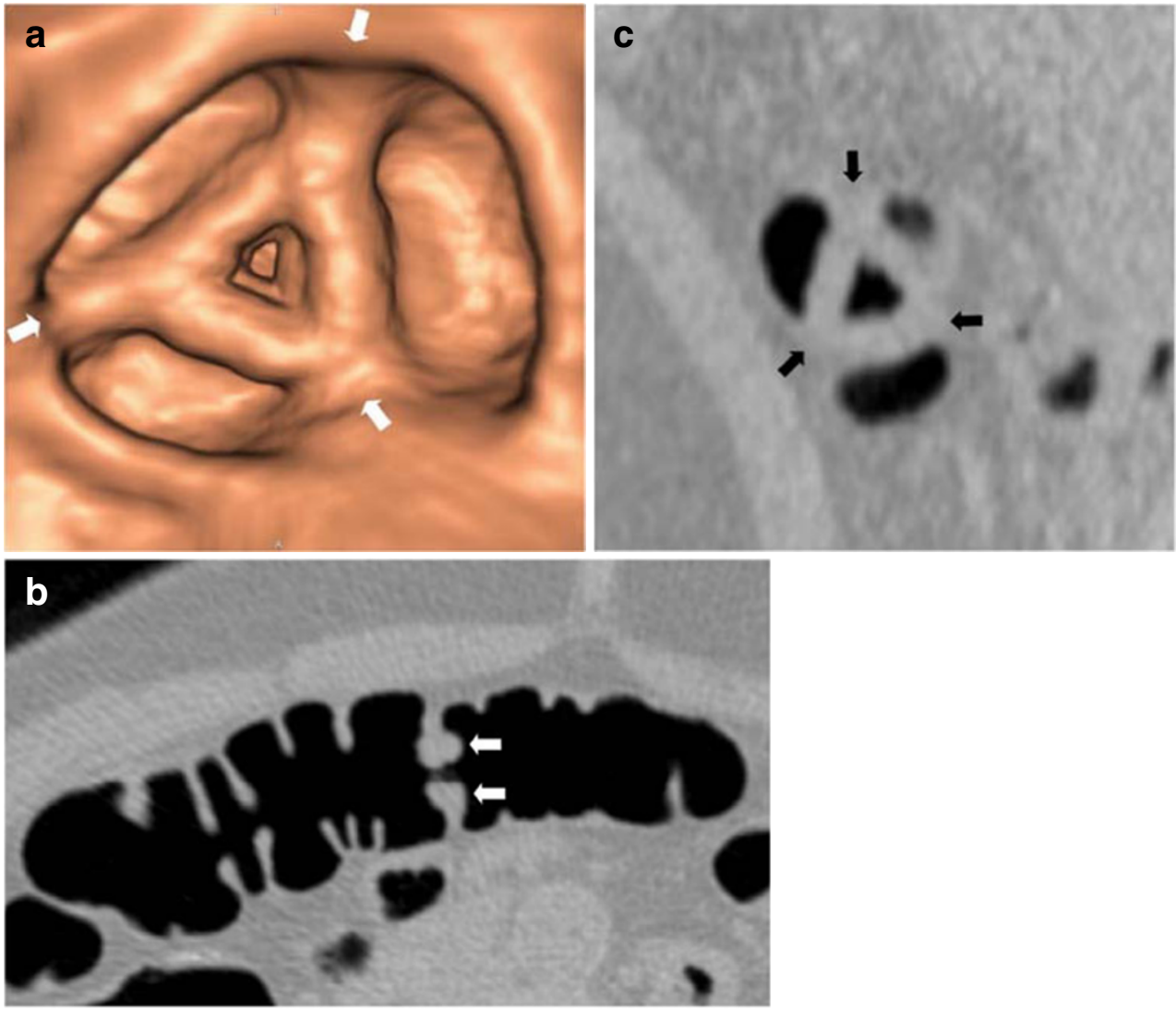

confirmed by detecting fatty tissue in the abdominal window setting or by checking its density (-100 to $0 \mathrm{HU})$.

\section{- Papillary transformation}

With papillary transformation, the terminal ileum protrudes or invaginates into the IC valve. This is a normal functional status with the IC valve acting as a sphincter preventing reflux of colonic contents to the small bowel. The IC valve becomes more prominent and may appear as a polypoid or even a tumoural structure. Frequently the normal lip-like structure becomes distorted with the IC valve appearing as a large, more or less rounded structure with smooth borders, with folds converging to a central depression, representing the connection with the terminal ileum, when assessed en face (Fig. 15). In 2D coronal or sagittal images allow for depiction of the normal lip-like structure.

\section{- Tumoural transformation}

With tumoural transformation the IC valve enlarges and shows distortion of its normal structure. Differentiation from a normal papillary valve is not always straightforward and needs inspection in both 2D and 3D imaging modes. Sometimes infiltration of the pericolonic fat with or without lymph nodes is detected.
Fig. 15 a Axial image of the caecum: large $>1 \mathrm{~cm}$ polypoid structure (white arrow). b The corresponding 3D image shows the defect as a large structure protruding into the colonic lumen. En face assessment shows converging folds towards a central depression: typical image of papillary transformation of the IC valve (white arrow)
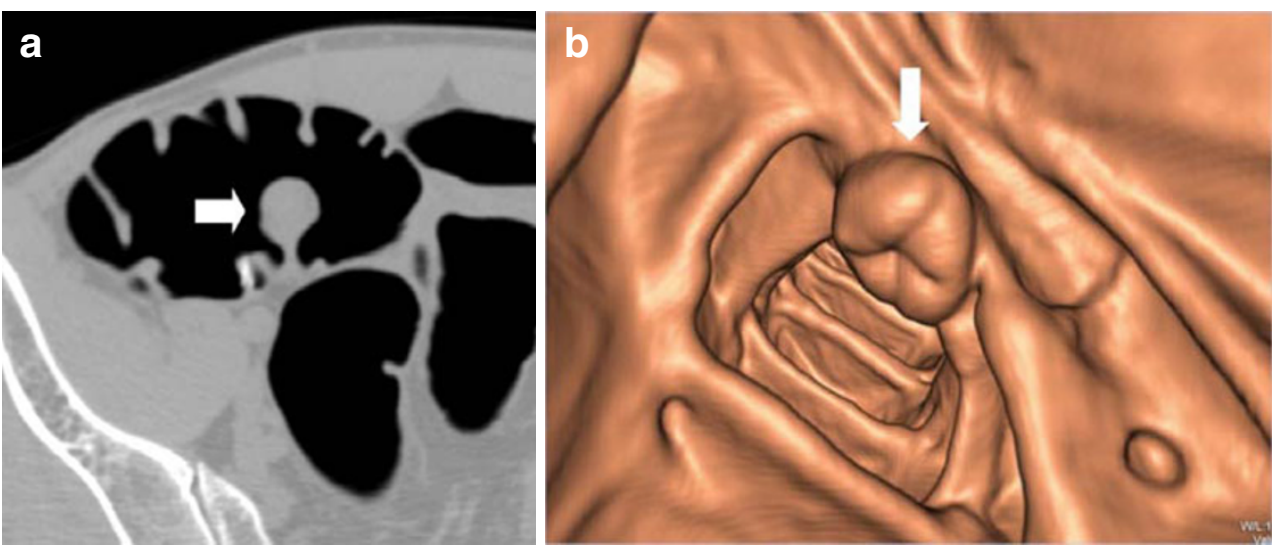
Appendix

The appendiceal orifice is situated at the convergence of the three taenia coli. It appears as a small opening frequently partly covered by a small fold. The appendix may prolapse into the colonic lumen. In this case it may appear as a small polypoid structure with a central depression representing the appendiceal orifice. Sometimes it appears as a small defect abutting the appendix in 2D. If the defect looks polypoid and exceeds $5 \mathrm{~mm}$, optical colonoscopy is necessary to exclude a significant tumoural lesion. An appendectomy stump may be another cause of a pseudopolypoid lesion in the caecum. CTC is not able to differentiate this entity from a regular polyp, necessitating optical colonoscopy whenever the defect exceeds $5 \mathrm{~mm}$.

\section{D and 3D imaging-related pitfalls}

2D-related pitfalls

\section{Polyp on a fold}

Polyps situated on a fold are particularly difficult to detect in 2D as sometimes they look like a normal extension of the semi-lunar fold. Three-dimensional CTC clearly shows the polyp prolapsing into the colonic lumen.

\section{The moving polyp}

A polyp with a long pedicle may undergo important positional shifts with dual positioning and mimic nontagged stool. Three-dimensional CTC clearly shows the pedunculated appearance of the luminal defect. Special care has to be taken if the pedicle is submerged by a contrast material pool.

\section{Polyp measurement}

Underestimation if the polyp size occurs when the detected polyp is only inspected on the axial images. Very frequently, the polyp appears larger on the coronal and sagittal images. This may change the polyp from a nonsignificant $(<6 \mathrm{~mm})$ to a significant $\geq 6 \mathrm{~mm}$ lesion, necessitating optical colonoscopy (Fig. 16).

3D-related pitfalls

\section{Polyp in fluid}

In $3 \mathrm{D}$ a polyp is of course invisible in fluid, while it is easy to detect as a negative filling defect in 2D (Fig. 2). Some software is provided with electronic cleansing removing the tagged residue in the colon. However this is not always a reliable tool and a polyp may be "cleansed" in the case of very dense tagging or insufficient cleansing may occur in the case of suboptimal tagging.

\section{Lesion characterisation}

A luminal defect detected in 3D always needs characterisation using $2 \mathrm{D}$ imaging. Indeed a $3 \mathrm{D}$ bump may be a true lesion. However, it may also correspond to residual stool, a diverticular faecalith, a lipoma and other entities as described above. Two-dimensional imaging using abdominal window settings reveals the exact nature of the luminal defect.

\section{Prominent folds}

In the case of prominent semi-lunar folds the haustrations become deeper and are more difficult to inspect in 3D. This is particularly the case in the ascending and transverse
Fig. 16 a Axial image of the sigmoid revealing a sessile diminutive 4-mm polyp (white arrow). b The corresponding sagittal image shows that the identical lesion is larger and becomes significant as it measures $7 \mathrm{~mm}$ (white arrow). This finding should prompt optical colonoscopy
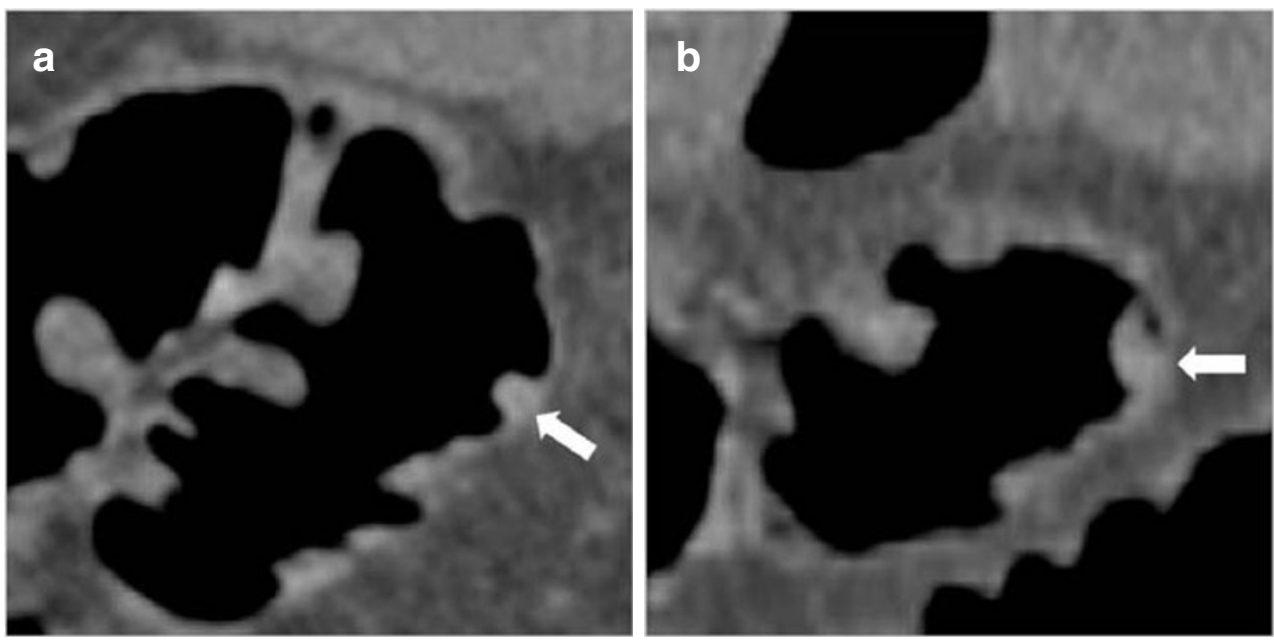
colon, and in the case of diverticulosis. Using 2D allows complete visualisation. Using 3D by turning the virtual camera between the folds, ensuring complete visualisation, is mandatory. Some software is provided with a missed region tool. This tool automatically shows the regions not covered during the regular fly-through in an antegrade and retrograde direction. Other software allows for alternative visualisation methods such as virtual pathological features depictinging the colon as if it were laid open showing all the hidden spots [14].

\section{Conclusion}

A flow chart for adequate CTC interpretation:

\section{Perform state-of-the-art CTC}

(a) Preparation based on faecal tagging

(b) Colonic distension with $\mathrm{CO}_{2}$ (+ injector), dual positioning and smooth muscle relaxation

2. General principles of interpretation

(a) Use the combined approach: 2D-3D

i. With a clean, well-distended colon: use 3D

ii. With failed preparation or suboptimal distension, diverticulosis: use 2D

(b) Always examine both supine and prone acquisitions

(c) Use all diagnostic tools: 3D, MPRs

(d) Use both the abdominal and intermediate window settings

3. Take special care when examining the rectum and caecum

\section{References}

1. Johnson CD, Chen MH, Toledano AY et al (2008) Accuracy of CT colonography for detection of large adenomas and cancers. $\mathrm{N}$ Engl J Med 359:1207-1217

2. Kim DH, Pickhardt PJ, Taylor AJ et al (2007) CT colonography versus colonoscopy for the detection of advanced neoplasia. $\mathrm{N}$ Engl J Med 357:1403-1412

3. Regge D (2007) the IMPACT Study Group Investigators (2007) Accuracy of CT colonography in subjects at increased risk of colorectal carcinoma: a multicenter study on 1000 patients. Presented at annual meeting of the Radiological Society of North America, Chicago
4. Graser A, Stieber P, Nagel D, Schafer C, Horst D, Becker CR et al (2009) Comparison of CT colonography, colonoscopy, sigmoidoscopy and faecal occult blood tests for the detection of advanced adenoma in an average risk population. Gut 58 (2):241-248

5. Burling D, International collaboration for CT colonography standards (2010) CT colonography standards. Clin Radiol 65:474-480

6. Rockey DC, Paulson E, Niedzwiecki D, Davis W, Bosworth HB, Sanders L et al (2005) Analysis of air contrast barium enema, computed tomographic colonography, and colonoscopy: prospective comparison. Lancet 365(9456):305-311

7. Doshi T, Rusinak D, Halvorsen RA, Rockey DC, Suzuki K (2007) Dachman AH (2005) CT colonography: false-negative interpretations. Radiology 244(1):165-173

8. Barish MA, Soto JA, Ferrucci JT (2005) Consensus on current clinical practice of virtual colonoscopy. AJR Am J Roentgenol 184:786-792

9. Lefere P, Gryspeerdt S, Dewyspelaere J et al (2002) Dietary fecal tagging as a cleansing method before CT colonography: initial results polyp detection and patient acceptance. Radiology 224:393-403

10. O'Connor SD, Summers RM, Choi JR et al (2006) Oral contrast adherence to polyps on CT colonography. J Comput Assist Tomogr 30:51-57

11. Pickhardt PJ, Kim DH, Taylor AJ (2008) Asymptomatic pneumatosis at CT colonography: a benign self-limited imaging finding distinct from perforation. AJR Am J Roentgenol 190:W112-W117

12. Yoshida H, Näppi J (2007) CAD in CT colonography without and with oral contrast agents: progress and challenges. Comput Med Imaging Graph 31:267-284

13. Chen JC, Dchman AH (2006) Cecal mobility: a potential pitfall of CT colonography. AJR Am J Roentgenol 186:1086-1089

14. Dachman AH, Lefere P, Gryspeerdt S, Morrin M (2007) CT colonography: visualization methods, interpretation, and pitfalls. Radiol Clin North Am 45:347-59

15. Reeders JW, Tytgat GN (1994) Clinical Radiology and endoscopy of the colon. Thieme, Stuttgart, pp 375-377

16. Gray H (1918) Gray's anatomy. Anatomy of the human body. XI Splanchnology, 2H The Large Intestine 1918. The Bartleby project at http://www.bartleby.com/107/249.html

17. Floch HM, Floch NR, Kowdley K et al (2005) Netter's Gastroenterology. V. Colon, rectum and anus. Icon Learning Systems, Teterboro

18. Gryspeerdt SS, Herman MJ, Baekelandt MA et al (2004) Supine/ left decubitus scanning: a valuable alternative to supine/prone scanning in CT colonography. Eur Radiol 14:768-777

19. Lefere P, Gryspeerdt S, Baekelandt M et al (2003) Diverticular disease in CT colonography. Eur Radiol 13:L62-L74

20. Glick SN (1991) Inverted colonic diverticulum: air contrast barium enema findings in six cases. AJR Am J Roentgenol 156:961-964

21. Chintapalli KN, Chopra S, Ghiatas AA et al (1999) Diverticulitis vs colon cancer: differentiation with helical CT findings. Radiology 210:429-435

22. Kelly JK (1991) Polypoid prolapsing mucosal folds in diverticular disease. Am J Surg Pathol 15:871-878 